\title{
Two-phase model analysis of the effects of interstimulus interval and masking task in human aversive classical conditioning*
}

\author{
WILLIAM F. PROKASY, WILLIAM C. WILLIAMS, WILLIAM Y. M. LEE, and KAROL L. KUMPFER \\ University of Utah, Salt Lake City, Utah 84112
}

\begin{abstract}
Three levels $(.5,1.0$, and $2.0 \mathrm{sec})$ of interstimulus interval (ISI) and presence vs absence of a masking task were manipulated in a human eyelid reflex conditioning situation. Through the use of a two-phase model of conditioning performance, it was concluded that (1) increasing the ISI and introducing a masking task increased the duration of Phase 1, (2) as ISI increased, the amount of conditioning decreased, (3) the trial-by-trial rate of change in response probability increased as a function of ISI, and (4) the masking task reduced operator limits. It was also noted that the typical ISI function is composed of rate effects at short ISIs and conditioning limit effects at long ISIs and that the larger $\theta$ at larger ISIs does not follow from a model of the stimulus trace hypothesis.
\end{abstract}

It has been shown (Prokasy, 1972, in press) that a two-phase, two-operator linear model, a variant of one first described by Norman (1964), describes in considerable detail the conditioning performance of both rabbits and humans. It is a two-phase model in that during Phase 1 response probability remains constant and during Phase 2 it modifies. $P_{i}$, response probability on Trial 1, is expressed as:

$$
\begin{aligned}
& \text { (1) } P_{i}=P_{o}, i=1 \text { to } K \text {; } \\
& \text { (2) } P_{i}=P_{i-1}+\theta_{1}\left(\lambda_{1}-P_{i-1}\right), \\
& i=K+1 \text { to } N \text {, given a CR on Trial } i-1 ; \\
& \text { (3) } P_{i}=P_{i-1}+\theta_{2}\left(\lambda_{2}-P_{i-1}\right), \\
& i=K+1 \text { to } N \text {, given no } C R \text { in Trial } i-1 \text {; }
\end{aligned}
$$

where $\mathrm{K}$ is the trial on which $\mathrm{S}$ switches from Phase 1 to Phase $2 ; P_{0}$ is the base level of response probability prior to conditioning; $\theta$ is a growth parameter; and $\lambda$ is a limit. In Phase 2 , one operator applies to CR trials and the other applies to non-CR trials. As described by Prokasy (in press), the method of dealing with $S$ protocols (the sequence of CR and non-CR trials) permits individual-S estimates of the model's parameters. These estimates then become the dependent variables through which it is possible to interpret the effects of independent variable manipulation.

The two variables analyzed in this experiment are the interstimulus interval (ISI) and the masking task context. The ISI has been investigated with a variety of species and response measures (e.g., Gormezano \& Moore, 1969, p. 136), and it has been concluded that asymptotic performance first increases and then decreases as a function of ISI. Based upon group learning curves, it has also been concluded (e.g., Schneiderman \&

\footnotetext{
*Supported by NSF Grant GN-12688 to the senior author. In addition, help with the costs of parameter estimation was provided through support from the University of Utah Research Committee.
}

Gormezano, 1964) that the rate of acquisition decreases as a function of the distance between the effective ISI and an optimal ISI. Such a result would be expected theoretically from Anderson's trace formulation (Anderson, 1959) of Hull's theory of the stimulus trace (Hull, 1943, 1952). At an empirical level, it is difficult, if not practically impossible, to distinguish rate and limit effects. In addition, in many studies it is not clear that asymptotic performance has been achieved across all values of the ISI. The use of a formal model as an analytic tool eliminates both of these problems.

The employment of a task designed to obscure the conditioning situation and, thereby, reduce cognitive influences in skeletal reflex conditioning was introduced by Spence (1963). Masking tasks have had a marked influence upon acquisition performance (Ross, 1971): they sometimes reduce overall performance and they reduce the level of differential performance. The use of a formal model provides an opportunity to isolate more clearly the locus of the masking task effects.

\section{METHOD}

\section{Apparatus}

The apparatus for sensing and recording eyelid responses is described elsewhere (Prokasy, Ebel, \& Thompson, 1963).

The CS was a $1,000-\mathrm{Hz}$ tone of $90-\mathrm{dB}$ (A) intensity, lasting .5, 1.0 , or $2.0 \mathrm{sec}$, depending on the group. The UCS was a $50-\mathrm{msec}$ puff of nitrogen, sufficient to support a $150-\mathrm{mm}$ column of mercury, which was delivered to the Ss' right cornea at CS offset. A continuous background level of $75 \mathrm{~dB}$ (A) white noise was employed to mask extraneous sounds from the laboratory. Stimuli were programmed by Western Union tape transmitters. Interstimulus intervals and CS and UCS durations were controlled by Tektronix timers.

\section{Subjects}

The Ss were volunteers from introductory psychology classes at the University of Utah. Eighteen were lost through apparatus failure or $\mathrm{E}$ error.

\section{Masking Task}

The masking task was a series of pattern detection problems 
Table 1

Number of Ss in Each Assumption Category

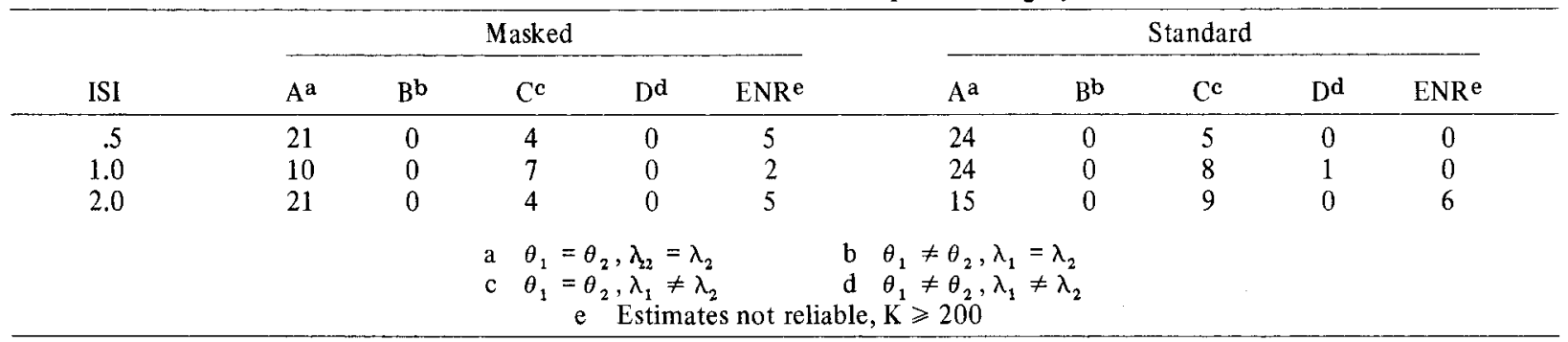

of increasing difficulty. A complete problem consisted of a sarticular sequence of 10 discrete left and right movements of a switch. The onset of a light for $2 \mathrm{sec}$ at fixed intervals of $5 \mathrm{sec}$ ignaled each left or right choice. An 18-mm-diam white jewel ight signaled the beginning of each new problem; $S$ was required o make the first left or right movement before the light went Jff. Each subsequent movement in the problem was signaled by in 18-mm-diam red jewel light. The onset of both lights ndicated that the last problem was being repeated. A counter abeled "number correct" incremented after each correct choice. $A$ second counter incremented within 2 sec regardless of whether Ir not a choice had been made or was correct. Both counters vere in full view of S. Simple single- and double-alternation roblems were presented early in the session, but more complex satterns were introduced as the session progressed.

\section{Experimental Design}

The design was a 2 by 2 orthogonal design with masking vs tandard instructions as one variable and ISI $(.5,1.0$, and $2.0 \mathrm{sec}$ ) as the other variable. The letters " $S$ " and " $M$ " will lesignate "standard" and "masking task" instructions, espectively, while the numbers $.5,1$, and 2 will designate ISI. Thus, Group .5M will refer to the group which received a $.5-\mathrm{sec}$ SI and the masking task condition. Ss were assigned Insystematically to the six groups, with final group Ns provided n Table 1.

Ss in all groups were exposed to the same sequence of daptation and conditioning trial stimuli, but the instructional ets differed. For Ss in the standard instruction groups, the nstructional set was to sit quietly, to let their reactions take care If themselves, and not to try to figure out the experiment. The nasking task Ss were told that the conditioning trials constituted timulation designed to distract them from the problem-solving ask.

The training trials were presented in two stages with no break retween the two stages. During the first stage, 60 unpaired trials, omposed of $30 \mathrm{CS}$ and 30 UCS trials, were presented at an verage intertrial interval of $6 \mathrm{sec}$. During the second stage, Ss eceived $250 \mathrm{CS}-\mathrm{UCS}$ pairings at an average intertrial interval of 0 sec. Since the value of the intertrial intervals varied in 1 -sec teps from 6 to $14 \mathrm{sec}$, there was no fixed relationship between he masking task trials (fixed intertrial interval of $5 \mathrm{sec}$ ) and the onditioning trials.

A CR was defined as a deflection of at least $1 \mathrm{~mm}$ or greater, alling in the latency range from $148 \mathrm{msec}$ after CS onset until $14 \mathrm{msec}$ after UCS onset. Thus, the recording intervals of the hree ISIs lasted (beginning $148 \mathrm{msec}$ after CS onset) for 376 , :76, and $1,376 \mathrm{msec}$, respectively. The pen deflection of $1 \mathrm{~mm}$ orresponds approximately to a .9 -mm eyelid movement.

\section{RESULTS}

With the exception of $P_{o}$, best-fit parameters were btained with the use of the computer subroutine iTEPIT (Chandler, 1969). $\mathrm{P}_{\mathrm{o}}$ was estimated empirically from responses to the $\mathrm{CS}$ during the preconditioning adaptation trials. Procedural details are provided by Prokasy (in press).

Best-fit parameters were obtained separately under four different assumptions:

$$
\begin{aligned}
& \text { (A) } \theta_{1}=\theta_{2} ; \lambda_{1}=\lambda_{2} \\
& \text { (B) } \theta_{1} \neq \theta_{2} ; \lambda_{1}=\lambda_{2} \\
& \text { (C) } \theta_{1}=\theta_{2} ; \lambda_{1} \neq \lambda_{2} \\
& \text { (D) } \theta_{1} \neq \theta_{2} ; \lambda_{1} \neq \lambda_{2}
\end{aligned}
$$

Since the model implied by Assumption A is a submodel of that implied by Assumptions B and C, and since the latter two are submodels of that implied by Assumption D, it was possible to employ a maximum-likelihood ratio test to determine which assumption was sufficient to account for the data (see Theios, 1968). Thus, for a given S, the likelihood (L) of the protocol was obtained separately under Assumptions $A$ and $B$, and a chi square was calculated as:

$$
\chi^{2}(1) \approx-2 \log _{\mathrm{e}} \frac{\mathrm{L}_{\mathrm{A}}}{\mathrm{L}_{\mathrm{B}}}
$$

The one degree of freedom reflects the fact that there is a one-parameter difference between the two assumptions. As an arbitrary procedure, the value of chi square (with $1 \mathrm{df}$ ) had to equal or exceed 4.0 before $\mathrm{B}$ could be chosen over $\mathrm{A}$ as the appropriate assumption. A similar strategy was employed for other comparisons, though it is to be noted that since $\mathrm{B}$ and $\mathrm{C}$ are not submodels of one another, choices had to be made indirectly through comparisons with Assumptions A and D. The method is described further elsewhere (Prokasy, in press).

The number of Ss in each treatment condition for whom a particular assumption was sufficient is provided in Table 1. Also included is the number of Ss for whom $K \geqslant 200$. For all practical purposes, these latter $\mathrm{Ss}$ did not condition or conditioned too late to permit a reliable estimate of parameters. The most obvious fact of 
Table 1 is that Assumptions A and C, but not B or D (with the exception of one S), were sufficient. This pattern, a large number of $\mathrm{Ss}$ consistent with Assumption $\mathrm{A}$ and a smaller number consistent with Assumption $\mathrm{C}$, did not vary reliably as a function either of ISI or of presence vs absence of masking task. The only reliable effect ${ }^{1}$ in Table 1 , aside from the large number of Ss categorized under Assumption A, is that, combined across both standard and masking contexts, the number of Ss not conditioning varies as a function of ISI, $\chi^{2}(2)=7.0$ with the largest number associated with the longest ISI.

Table 2 provides the obtained mean parameter estimates for each group. Note that the $P_{o}$ and $K$ values include the Ss who did not condition, and that the $\theta$ values combine Ss under both Assumptions $A$ and $C$. As required by the different assumptions, $\lambda$ is provided for Assumption A Ss, while both $\lambda_{1}$ and $\lambda_{2}$ are provided for Assumption C Ss. The Ns for these means can be obtained from Table 1.

In all groups, $\lambda_{1}>\lambda_{2}$. For those conditioned with the masking task, 13 of the 15 Ss described by Assumption C yielded $\lambda_{1}>\lambda_{2}$. Similarly, of the $22 \mathrm{Ss}$ in the normal context groups described by Assumption $C$, 20 yielded $\lambda_{1}>\lambda_{2}$. There were no differences in categorization frequencies as a function of either independent variable.

As would be expected, $P_{o}$ increased with ISI, $F(2,168)=21.23$, but no other effect was significant. Rather dramatic effects of the two independent variables were obtained, however, on $\mathrm{K}$. $\mathrm{K}$ was larger in the presence of the masking task, $F(1,168)=16.33$. While there was no direct effect of ISI, the interaction between ISI and context reveals quite clearly that $\mathbf{K}$ remained relatively constant across ISI with the masking task, but increased with ISI in the standard context, $F(2,168)=$ 5.33 . With the 2 -sec ISI, there was no difference in $\mathrm{K}$ between the standard and masking task context Ss.

To analyze $\theta$ factorially, cell Ns were made proportional by selecting randomly enough $\mathrm{Ss}$ from the appropriate groups to yield $25 \mathrm{Ss}$ each in Groups $2 \mathrm{M}$ and $2 \mathrm{~S}$. The conditioning context had no effect on $\theta$, $\mathrm{F}(1,142)=.30$, but $\theta$ increased as a direct function of ISI, $F(2,142)=3.71$. The increase was primarily between ISIs of .5 and 1.0. The numerical value of $\theta$ was

Table 2

Average Best-Fit Parameter Estimates for Ss Falling in Each of the Two Major Assumption Categories

\begin{tabular}{|c|c|c|c|c|c|c|}
\hline \multirow[b]{2}{*}{ Group } & \multicolumn{3}{|c|}{ Combined Ss } & \multirow{2}{*}{$\begin{array}{c}\text { Assump- } \\
\text { tion } A \\
\lambda \\
\end{array}$} & \multicolumn{2}{|c|}{ Assumption $\mathrm{C}$} \\
\hline & $P_{0}$ & $\mathrm{~K}$ & $\theta$ & & $\lambda_{1}$ & $\lambda_{2}$ \\
\hline $\begin{array}{l}.5 \mathrm{M} \\
1 \mathrm{M} \\
2 \mathrm{M}\end{array}$ & $\begin{array}{l}.129 \\
.298 \\
.483\end{array}$ & $\begin{array}{l}63.2 \\
71.0 \\
48.2\end{array}$ & $\begin{array}{l}.469 \\
.773 \\
.734\end{array}$ & $\begin{array}{l}.496 \\
.438 \\
.529\end{array}$ & $\begin{array}{l}.558 \\
.675 \\
.780\end{array}$ & $\begin{array}{l}.340 \\
.202 \\
.616\end{array}$ \\
\hline $\begin{array}{l}.5 \mathrm{~S} \\
1 \mathrm{~S} \\
2 \mathrm{~S}\end{array}$ & $\begin{array}{l}.101 \\
.205 \\
.424\end{array}$ & $\begin{array}{l}18.3 \\
30.6 \\
45.4\end{array}$ & $\begin{array}{l}.381 \\
.704 \\
.684\end{array}$ & $\begin{array}{l}.750 \\
.483 \\
.624\end{array}$ & $\begin{array}{l}.648 \\
.595 \\
.750\end{array}$ & $\begin{array}{l}.356 \\
.429 \\
.233\end{array}$ \\
\hline
\end{tabular}

Table 3

Correlation Between $P_{0}$ and $K$ and Average $P_{0}$ and $P_{k}$

\begin{tabular}{ccccc}
\hline Group & $\mathrm{R}_{\mathrm{P}_{\mathrm{O}}, \mathrm{K}}$ & $\mathrm{P}_{\mathrm{O}}$ & $\mathrm{P}_{\mathrm{K}}$ & $\mathrm{N}$ \\
\hline $.5 \mathrm{M}$ & .237 & .129 & .167 & 30 \\
$1 \mathrm{M}$ & -.162 & .298 & .286 & 28 \\
$2 \mathrm{M}$ & .073 & .483 & .600 & 30 \\
$.5 \mathrm{~S}$ & -.167 & .101 & .207 & 29 \\
$1 \mathrm{~S}$ & -.053 & .205 & .303 & 33 \\
$2 \mathrm{~S}$ & $.324^{*}$ & .424 & .500 & 30 \\
\hline
\end{tabular}

${ }^{*} p<.05$

quite high at the longer ISIs, resulting from the fact that a total of 62 of the $107 \mathrm{Ss}$ in the four longer ISI groups had $\theta=1.0$. For these $S s$ the performance shifts between $\mathrm{P}_{\mathrm{o}}$ and $\lambda$ (Assumption A) or between $\lambda_{1}$ and $\lambda_{2}$ (Assumption C) were all or none. In the case of the Assumption C Ss, shifts were made following CR and non-CR trials.

The only reliable effect of either of the variables on $\lambda$ was that, for the Assumption A Ss, the masking task resulted in a lower value, $F(1,122)=5.61$. It is to be emphasized, though, that the lack of an effect of ISI on $\lambda$ does not imply no effect on level of conditioning. With the obvious effect on $P_{o}$ (since the measuring interval expands with ISI), the distance between $P_{o}$ and $\lambda$ or $\lambda_{1}$ does decrease as a function of ISI.

Some, though limited, information on the role of initial response levels on the transfer from Phase 1 to Phase 2 can be obtained from the present data. Table 3 presents the correlations between $\mathrm{P}_{\mathrm{o}}$ and $\mathrm{K}$. Of the six correlations, three are slightly positive and three slightly negative, with one of the positive correlations reliable. There is, therefore, no evidence of a consistent relationship. A further statistic of interest is the difference between $P_{o}$ and $P_{k}$, and these data are also provided in Table 3. There is some evidence, particularly in those $S s$ trained under the standard instruction conditions, that $P_{k}>P_{o}$, though the difference is not reliable unless the data are pooled across groups. What is clear is that there is no dominant trend for $\mathbf{P}_{k}$ to approach either zero or unity, i.e., for the transition from Phase 1 to Phase 2 to be correlated either with the presence or the absence of a CR on Trial K. Thus, neither initial response rate nor Trial $\mathrm{K}$ performance is strongly associated with the Phase 1 to Phase 2 transition.

\section{DISCUSSION}

\section{Comparison with Past Research}

The two-phase model was examined under four different assumptions: the four different combinations possible with $\theta$ s equal or unequal and $\lambda s$ equal or unequal. Consistent with past research on humans and rabbits (Prokasy, in press), the Ss fell, with one exception, into one of two assumption categories. One group, by far the larger, required but a single operator 
during Phase 2 to describe performance. For these Ss, performance remained constant during Phase 1 and then increased monotonically on a trial-by-trial basis toward a limit. Only four parameters are required to describe their protocols: $\mathbf{P}_{\mathbf{o}}, \mathrm{K}, \theta$, and $\lambda$.

The remaining $S s$ required two operators during Phase 2. Since $\theta_{1}=\theta_{2}$ for these Ss, only the addition of a single parameter was required (i.e., dividing $\lambda$ into $\lambda_{1}$ and $\lambda_{2}$ ) over the one operator version. As is the case in past observations (Prokasy, in press), $\lambda_{1}>\lambda_{2}$, which means that, once it exceeds $\lambda_{2}, P_{i}$ increases following $\mathrm{CR}$ trials and decreases following non-CR trials.

The consistency of present outcomes with those of the earlier research implies not only common classes of outcome across species, but common classes across various levels of independent variable manipulation. It illustrates, too, that, within a single model class, an increase in the number of parameters required to describe the data is not arbitrary: in going from four to five parameters, the uniform outcome has been that the additional $\lambda$, not $\theta$, extracts additional reliable information from the data.

\section{Initial Acquisition}

As Gormezano and Moore (1969) point out, it is not clear from a reinforcement point of view how initial acquisition is effected in classical conditioning. One reinforcement interpretation (Spence, 1956) is that the pairing of CS and UCS results in an increment in habit strength, this increasing gradually to a point which yields above-baseline levels of responding. While the existence of Phase 1 is consistent with such a view, the Assumption C Ss (i.e., those who exhibited a decrease in response level following non-CR trials) do not perform in a manner consistent with the theory. If below-threshold increments in habit strength are occurring during Phase 1 independent of CR presence or absence (as the Spence model suggests), then it is difficult to understand why the failure to make a CR during Phase 2 would yield decrements in response probability.

A possible refinement is that reinforcement occurs only when the CR occurs, even during Phase 1 when chance responding will, eventually, yield a temporally appropriate response. If this were the case, one would anticipate a high negative correlation between $P_{o}$ and $K$ and also a high response probability on Trial $K$, following which transition to Phase 2 occurs. No evidence for the former was obtained, and the limited positive evidence for the latter suggests, at best, a small effect.

\section{Interstimulus Interval}

As anticipated, based upon earlier research (Gormezano \& Moore, 1969, p. 36), the distance between $P_{o}$ and $\lambda$, i.e., the amount of conditioning, decreased as a function of ISI. Not anticipated is the fact that the slow rate of acquisition associated with the longer ISIs is a function of increased $\mathrm{K}$, not reduced $\theta$. The rate of change in response probability once Phase 2 is entered is greater with longer ISIs.

That $\theta$ increased as a direct function of ISI was not only unexpected, it is the opposite of what would be expected based upon Anderson's model (Anderson, 1959) of Hull's theory of the stimulus trace. In addition, it is to be noted that those Ss with $\theta=1.0$ are not functioning in typical incremental-decremental fashion. Their behavior approximates that which might be expected from a Markov process (Theios, 1971).

As ISIs increase from 0.0 through $2.5 \mathrm{sec}$ in eyelid conditioning, level of conditioning is reported as a concave downward function, with a maximum near 500 msec. Since $\mathrm{K}$ is shorter and $\lambda-\mathrm{P}_{\mathrm{o}}$ greater at short ISIs, the present analysis suggests that the function results from reductions in $\theta$ at short ISIs and reductions in amount of conditioning at long ISIs. An empirical analysis of this possibility will be hindered, however, by the fact that latency distributions are incomplete at short ISIs (e.g., Ebel \& Prokasy, 1963).

\section{Masking Task}

One of the objectives of this study was to isolate the effects of introducing a masking task during conditioning. The effects were limited to an increase in $\mathrm{K}$ and a decrease in $\lambda$ for the Assumption A Ss. The failure to shift the number of $\mathrm{Ss}$ for whom Assumption $A$ is sufficient, to have any effect on the ordering of the magnitude of $\lambda_{1}$ or $\lambda_{2}$, or to alter the pattern of parameters in any significant way indicated that the masking task had relatively elementary effects on performance.

The interaction between ISI and the masking task with $\mathrm{K}$ as the dependent variable shows that, for all practical purposes, the masking task had no differential effects at the 2 -sec ISI. It is possible that at the shorter ISIs $S$ did not always detect the CS. This seems unlikely, however, since one would then anticipate both a reduction in $\theta$ and a reduction in the number of two-operator Ss in the masking task groups, neither of which occurred. A clearer test of this would involve the use of a masking task in which $S$ is required to make a differential motor response to CS onset (Ross, Wilcox, \& Mayer, 1967), thereby insuring that $S$ did, in fact, detect the CS.

\section{REFERENCES}

Anderson, N. H. Temporal properties of response evocation. In R. R. Bush and W. K. Estes (Eds.) Studies in mathematical learning theory. Stanford: Stanford University Press, 1959. Pp. 125-134.

Chandler, J. P. STEPIT: Finds local minima of a smooth function of several parameters. (CPA 312) Behavioral Science, $1969,14,81-82$. 
Ebel, H. C., \& Prokasy, W. F. Classical eyelid conditioning as a function of sustained and shifted interstimulus intervals. Journal of Experimental Psychology, 1963, 65, 52-58.

Gormezano, I., \& Moore, J. W. Classical conditioning. In M. H. Marx (Ed.), Learning: Processes. London: MacMillian, 1969. Pp. 121-203.

Hull, C. L. Principles of behavior: An introduction to behavior theory. New York: Appleton-Century-Crofts, 1943.

Hull, C. L. A behavior system. New Haven: Yale University Press, 1952.

Norman, M. F. A two-phase model and an application to verbal discrimination learning. In R. C. Atkinson (Ed.), Studies in mathematical psychology. Stanford: Stanford University Press, 1964. Pp. 173-187.

Prokasy, W. F. Developments with the two-phase model applied to human eyelid conditioning. In $A$. H. Black and W. F. Prokasy (Eds.), Classical conditioning II. New York: Appleton-Century-Crofts, 1972. Pp. 119-147.

Prokasy, W. F. A two-phase model account of aversive classical conditioning performance in humans and rabbits. Learning \& Motivation, in press.

Prokasy, W. F., Ebel, H. C., \& Thompson, D. D. Response shaping at long interstimulus intervals in classical eyelid conditioning. Journal of Experimental Psychology, 1963, 66, 138-141.

Ross, L. E. Cognitive factors in conditioning: The use of masking tasks in eyelid conditioning. In H. H. Kendler and J. T. Spence (Eds.), Essays in neobehaviorism. New York: Appleton-Century-Crofts, 1971. Pp. 161-185.

Ross, L. E., Wilcox, S. M., \& Mayer, M. J. A simple eyelid conditioning masking task and its effects on differential conditioning. Psychonomic Science, 1967, 9, 333-334.
Schneiderman, N., \& Gormezano, I. Conditioning of the nictitating membrane of the rabbit as a function of CS-US interval. Journal of Comparative \& Physiological Psychology, 1964, 57, 188-195.

Spence, K. W. Behavior theory and conditioning. New Haven: Yale University Press, 1956.

Spence, K. W. Cognitive factors in the extinction of the conditioned eyelid response in humans. Science, 1963, 140, 1224-1225.

Theios, J. Finite integer models for learning in individual subjects. Psychological Review, 1968, 75, 292-307.

Theios, J. Mathematical models for aversive conditioning. In $\mathrm{R}$. R. Brush (Ed.), Aversive conditioning and learning. New York: Academic Press, 1971. Pp. 297-346.

\section{NOTES}

1. Unless stated otherwise, the .05 rejection region was adopted in all statistical tests.

2. Since $K$ is only an estimate of the point of transition between Phases 1 and 2, it may be smaller or larger than the parameter. If larger, then $\mathbf{P}_{\mathbf{k}}$ would be biased upward. If smaller, no effect would be obtained (i.e., response probability is stationary before Trial $K+1$ ). A pooling of estimates, some of which are high and others of which are low, will, therefore, yield a bias for $P_{k}$ to be greater than $P_{0}$. Whether or not this accounts for the trrend in Table 3 cannot be decided on the basis of present data.

(Received for publication May 29, 1973; revision received July 20,1973 .) 